\title{
Carbon-capability framework for Malaysia: towards encouraging low-carbon community lifestyles
}

\begin{abstract}
Human welfare is increasingly affected by the climate change impacts where more scientific evidence has pointed to a significant human contribution as the most contributing factors. Lately, the concept of 'carbon-capability' has been proposed recently to get the relative meanings related to carbon and individual power and enthusiasm to cut emissions. Thus, this paper aims to present a review of the theoretical framework and identifies the need for more specific components underlying the community carbon-capability. This study analyzes by using content analysis and based in part on related earlier study. The paper concludes that community carbon-capability framework's ability to help in reducing climate change impacts and highlighted the vital to understanding community engagement with overall climate change issues in Malaysia.
\end{abstract}

Keyword: Community engagement; Climate change; Carbon-capability; Low-carbon community 